\title{
ERK2 phosphorylation of serine 77 regulates Bmf pro-apoptotic activity
}

\author{
Y Shao ${ }^{1}$ and AE Aplin*,1,2
}

B-cell lymphoma 2 (Bcl-2) homology 3 (BH3)-only proteins represent a class of pro-apoptotic factors that neutralize pro-survival $\mathrm{Bcl}-2$ proteins, and, in some cases, directly activate Bax. The mechanisms of control and the role of BH3-only proteins, such as $\mathrm{Bcl}-2$ like protein 11 extra large and Bad are well studied. By contrast, relatively little is known about the regulation and role of $\mathrm{Bcl}-2$ modifying factor (Bmf). The B-RAF oncogene is mutated in $\sim 8 \%$ of human tumors. We have previously shown that Bmf is upregulated at the transcript level and is required for apoptosis induced by targeting B-RAF signaling in tumor cells harboring mutant B-RAF. In this study, we show that Bmf is regulated at the post-translational level by mutant B-RAF-MEK-ERK2 signaling. Extracellular signal-regulated kinase (ERK2) directly phosphorylates Bmf on serine 74 and serine 77 residues with serine 77 being the predominant site. In addition, serine 77 phosphorylation reduces Bmf pro-apoptotic activity likely through a mechanism independent of altering Bmf localization to the mitochondria and/or interactions with dynein light chain 2 and the pro-survival proteins, B-cell lymphoma extra large, Bcl-2 and Mcl-1. These data identify a novel mode of regulation in Bmf that modulates its pro-apoptotic activity in mutant B-RAF tumor cells.

Cell Death and Disease (2012) 3, e253; doi:10.1038/cddis.2011.137; published online 19 January 2012

Subject Category: Cancer

B-cell lymphoma 2 (Bcl-2) homology 3 (BH3)-only proteins represent a class of pro-death regulators in the canonical intrinsic mitochondrial apoptotic pathway. Outside of the $\mathrm{BH} 3$ domain, the overall homology varies considerably between members of this subfamily. They act through the neutralization of pro-survival proteins (such as Bcl-2, B-cell lymphoma extra large (Bcl-xL), Bcl-2 like protein $2(\mathrm{Bcl}-\mathrm{w})$ and $\mathrm{Mcl}-1)$, which in turn repress the essential pro-apoptotic proteins, Bax and Bak. In addition, some BH3-only proteins, including Bim, caspase-activated $\mathrm{tBid}$, and possibly other $\mathrm{BH}$-only proteins, may directly activate Bax. ${ }^{1-3}$ Activation of Bax and Bak leads to mitochondrial outer membrane permeabilization and the release of mitochondrial factors, such as cytochrome C, Smac, apoptosis-inducing factor. These factors promote the initiation of the caspase cleavage cascade, as well as caspase-independent forms of programmed cell death. ${ }^{4,5}$

The BH3-only protein, $\mathrm{Bcl}-2$ modifying factor (Bmf), was originally identified through a yeast-2-hybrid screen with Mcl-1 as the bait. ${ }^{6}$ Subsequent reports showed that Bmf also strongly interacts with $\mathrm{Bcl}-2, \mathrm{Bcl}-\mathrm{xL}$ and $\mathrm{Bcl}-\mathrm{w} .{ }^{7,8} \mathrm{Bmf}$ is critical for apoptosis induced by various stimuli in multiple cell types. Bmf-/- lymphocytes are resistant to apoptosis induced by glucocorticoids or histone deacetylase inhibition. ${ }^{9}$ Relevant to tumor biology, knockout of Bmf increases tumor load in the $\mathrm{E} \mu$-myc transgenic mouse model of $\mathrm{B}$-cell lymphoma. ${ }^{10}$ We have recently shown a role for $\mathrm{Bmf}$ in apoptosis induced following oncogene targeting in melanoma. B-RAF is mutated in $40-60 \%$ of melanomas. Mutant B-RAF melanoma cells are susceptible to apoptosis in 3D collagen when B-RAF is targeted and this process is dependent on upregulation of $\mathrm{Bmf}$, as well as $\mathrm{Bcl}-2$ like protein 11 extra large (Bim-EL). ${ }^{11}$

Early studies suggested that Bmf interacts with the actinbased myosin $\mathrm{V}$ motor complex via dynein light chain (DLC)2, which sequesters Bmf to the cytoskeleton under healthy conditions. ${ }^{6}$ Upon certain cellular stress conditions, such as UV radiation or loss of extracellular matrix adhesion, Bmf is released from DLC2 and relocalizes to the mitochondria, where it elicits pro-apoptotic activity. ${ }^{12,13}$ In addition to alterations in its subcellular localization, Bmf is also regulated at the transcript level. We and others have observed upregulation of Bmf mRNA level following inhibition of B-RAF-MEK-ERK pathway in melanoma cells, although further mechanistic details remain elusive. ${ }^{11,14}$

Significant control of BH3-only protein activity is exerted at the post-translation level. In this regard, the control of Bmf is understudied compared with other BH3-only proteins. Bim-EL and Bad are known to be phosphorylated by extracellular signal-regulated kinase (ERK) $1 / 2,{ }^{15-17} \mathrm{c}$-Jun $\mathrm{N}$-terminal kinase $(\mathrm{JNK})^{13,18,19}$ and p38 mitogen-activated

\footnotetext{
${ }^{1}$ Department of Cancer Biology, Kimmel Cancer Center, Thomas Jefferson University, 233 South 10th Street, Philadelphia, PA 19107, USA; ${ }^{2}$ Department of Dermatology and Cutaneous Biology, Thomas Jefferson University, 233 South 10th Street, Philadelphia, PA 19107, USA

*Corresponding author: AE Aplin, Department of Cancer Biology, Kimmel Cancer Center, Thomas Jefferson University, 233 South 10th Street, Philadelphia, PA 19107, USA. Tel: + 215503 7296; Fax: + 215923 9248; E-mail: Andrew.Aplin@KimmelCancerCenter.Org

Keywords: Bmf; phosphorylation; melanoma; ERK2

Abbreviations: Bcl-2, B-cell lymphoma 2; Bcl-w, Bcl-2 like protein 2; Bcl-xl, B-cell lymphoma extra large; BH3, Bcl-2 homology 3; Bim-EL, Bcl-2 like protein 11 extra large; Bmf, Bcl-2 modifying factor; CIAP, calf intestine alkaline phosphatase; DLC1/2, dynein light chain 1/2; Dox, doxycycline; ERK1/2, extracellular signal-regulated kinase 1/2; FBS, fetal bovine serum; FMK, fluoromethyl ketone; GST, glutathione S transferase; JNK, c-Jun N-terminal kinase; MAPK, mitogen-activated protein kinase; Mcl-1, myeloid cell leukemia sequence 1; MEK, mitogen-activated protein kinase kinase; RSK, ribosome S6 kinase; siRNA, small interference RNA; SP, serine-proline; TP, threonine-proline; WT, wild type

Received 20.9.11; revised 05.12.11; accepted 07.12.11; Edited by P Salomoni
} 
protein kinases (MAPKs) ${ }^{20}$ and by ribosomal S6 kinase 1 (RSK). ${ }^{21}$ Bmf phosphorylation by JNK has been demonstrated in vitro. ${ }^{13}$ The phosphorylation site was postulated to be serine 74 based on a similarity to a JNK phosphorylated site, serine 58, in Bim-L. ${ }^{13}$ Phosphomimetic mutation of this site (S74D) moderately enhanced Bmf apoptotic activity in vivo. ${ }^{22}$ Here, we demonstrate a previously unrecognized mode of regulation of Bmf. We show that B-RAF-MEK-ERK2 signaling regulates $\mathrm{Bmf}$ phosphorylation at serine 74 and serine 77. Phosphorylation of serine 77 downregulates the pro-apoptotic activity of Bmf.

\section{Results}

Bmf induces apoptosis independent of Bim-EL in melanoma cells. We have previously shown that targeting mutant B-RAF ${ }^{\mathrm{V} 600 E}$ using small interference RNA (siRNA) or the B-RAF inhibitor, PLX4720, induces apoptosis in primary melanoma cell lines that is mediated extensively by the upregulation of two BH3-only proteins, Bim-EL and Bmf. ${ }^{11}$ Although both Bim-EL and Bmf contributed to B-RAFtargeting-induced cell death, Bmf knockdown exhibited a stronger rescue effect than Bim-EL knockdown, ${ }^{11}$ indicating that Bmf may have a major role in this process. To test whether Bmf expression is sufficient to induce apoptosis, we generated five inducible melanoma cell lines (WM793TR/HABmf, Sbcl2TR/HA-Bmf, 1205LuTR/HA-Bmf, WM115TR/HABmf and A375TR/HA-Bmf) that expressed HA-tagged Bmf upon doxycyline treatment. Induction of $\mathrm{HA}-\mathrm{Bmf}$ was detected by western blot (Figure 1a) and all cell lines displayed increased apoptosis following $48 \mathrm{~h}$ of Bmf expression with the exception of A375TR/HA-Bmf (Figure 1b).

$A$ recent report showed that pacilitaxel-induced apoptosis occurs via a domino effect whereby Bmf (and p53-upregulated modulator of apoptosis) displace Bim from pro-survival Bcl-2 family proteins. ${ }^{23}$ To test whether Bmfinduced apoptosis in melanoma cells was dependent on Bim, we knocked down Bim in Bmf-induced cells. WM793TR/HABmf cells express low basal levels of Bim-EL because of its rapid turnover mediated by ERK phosphorylation. ${ }^{16-18}$ Treatment of Bim-specific siRNAs further reduced Bim-EL level (Figure 1c). Similar levels of $\mathrm{HA}-\mathrm{Bmf}$ were induced by doxycycline (Dox) in both control and Bim knockdown cells (Figure 1c). However, reduced Bim-EL expression failed to rescue Bmf-induced apoptosis (Figure 1d). These data suggest that Bmf expression is sufficient to induce cell death independent of Bim-EL.

\section{ERK2 signaling regulates the mobility of Bmf indicative of phosphorylation. When expressing Bmf in melanoma cells, we consistently observed a slower-migrating population of Bmf by SDS-PAGE (Figure 1a) indicating the presence of post-translational modification. Other BH3-only proteins, Bim-EL and Bad, are known to be phosphorylated by ERK1/2, ${ }^{15-17} \mathrm{JNK},{ }^{13,18,19} \mathrm{p} 38^{20}$ and RSK1. ${ }^{21}$ Treatment with calf intestine alkaline phosphatase (CIAP) reduced the slower-migrating Bmf population in Bmf immunoprecipitates from induced A375TR/HA-Bmf cells, an effect that was reversed with phosphatase inhibitors (Figure 2a). Thus, we}

tested for kinase signaling pathways that regulate Bmf by treating induced WM793TR/HA-Bmf cells with kinase inhibitors that target the RAF (PLX4720), MEK1/2 (U0126), p38 (SB203580), JNK (SP600125) or phosphatidylinositol 3 kinase (LY294002) pathways. The effect on the electrophoretic mobility of Bmf was analyzed. All these inhibitors efficiently blocked corresponding pathways, as demonstrated by western blotting of downstream cognate targets (Figure 2b). Treatment with the RAF inhibitor, PLX4720, or the MEK inhibitor, U0126, completely eliminated the electrophoretic mobility shift of Bmf, whereas SP600125 (JNK inhibitor) and LY294002 (phosphatidylinositol 3 kinase inhibitor) treatment showed no effect. The p38 inhibitor, SB203580, partially decreased the mobility shift of Bmf, and also partially reduced ERK $1 / 2$ signaling. These results suggested that Bmf may be phosphorylated by MEK-ERK1/ 2 and possibly p38 signaling in melanoma cells.

To further investigate the role of MAPKs in the Bmf mobility shift, we used specific siRNAs to knock down ERK1, ERK2 and p38 $\alpha$ individually in Dox-treated WM793TR/HA-Bmf cells. ERK2 but not ERK1 knockdown significantly reduced Bmf mobility shift comparable to U0126 treatment (Figure 2c). p38 $\alpha$ siRNA did not affect the mobility shift of Bmf (Figure 2c) but did decrease phospho-p38 levels (Figure 2d), suggesting that $p 38 \alpha$ is the major p38 isoform in WM793 cells. Therefore, ERK2, but not ERK1 or p38 signaling contributes to the mobility shift/phosphorylation of Bmf in melanoma cells. RSKs are downstream effectors of ERK signaling and have been shown to phosphorylate Bim-EL. ${ }^{21}$ Treatment with the RSK inhibitors, fluoromethyl ketone (FMK), ${ }^{24}$ failed to reduce Bmf mobility shift (Figure 2e), arguing against a direct role of RSKs in Bmf phosphorylation.

To further test whether ERK2 signaling can phosphorylate Bmf, we ectopically expressed HA-Bmf alone or in combination with wild-type ERK2 (ERK2 WT) or constitutive active ERK2 (ERK2 CA) in 293FT cells. The basal MEK/ERK pathway activity is low in 293FT cells and no apparent electrophoretic mobility shift was detected when Bmf was expressed alone or together with ERK2 WT (Figure 2f). Notably, co-expression of HA-Bmf and ERK2 CA significantly increased mobility shift of Bmf further supporting a role for activated ERK2 to phosphorylate Bmf.

ERK2 directly phosphorylates Bmf on serine 74 and serine 77. Serine-proline (SP) and threonine-proline (TP) motifs are most common targets of ERK2 phosphorylation. Scanning the Bmf protein sequences identified two phylogenetically conserved SP sites, serine 74 and serine 77 (Figure 3a), but no TP sites. To test these two candidate phosphorylation sites, we generated WM793TR cell lines that inducibly express $\mathrm{HA}-\mathrm{Bmf}$ with alanine substitutions at serine 74 (HA-Bmf S74A) or serine 77 (HA-Bmf S77A) or both serine 74 and serine 77 (HA-Bmf $A A)$. Replacement of individual serine residue at serine 74 or serine 77 with alanine partially reduced the electrophoretic mobility shift of Bmf, whereas a double mutation completely eliminated the mobility shift (Figure 3b). Loss of serine 77 had a stronger impact on the mobility shift of Bmf than loss of serine 74 . Conversely, replacement of serine 74 or serine 77 with the phosphomimetic residue, aspartic acid (S74D or S77D), was 

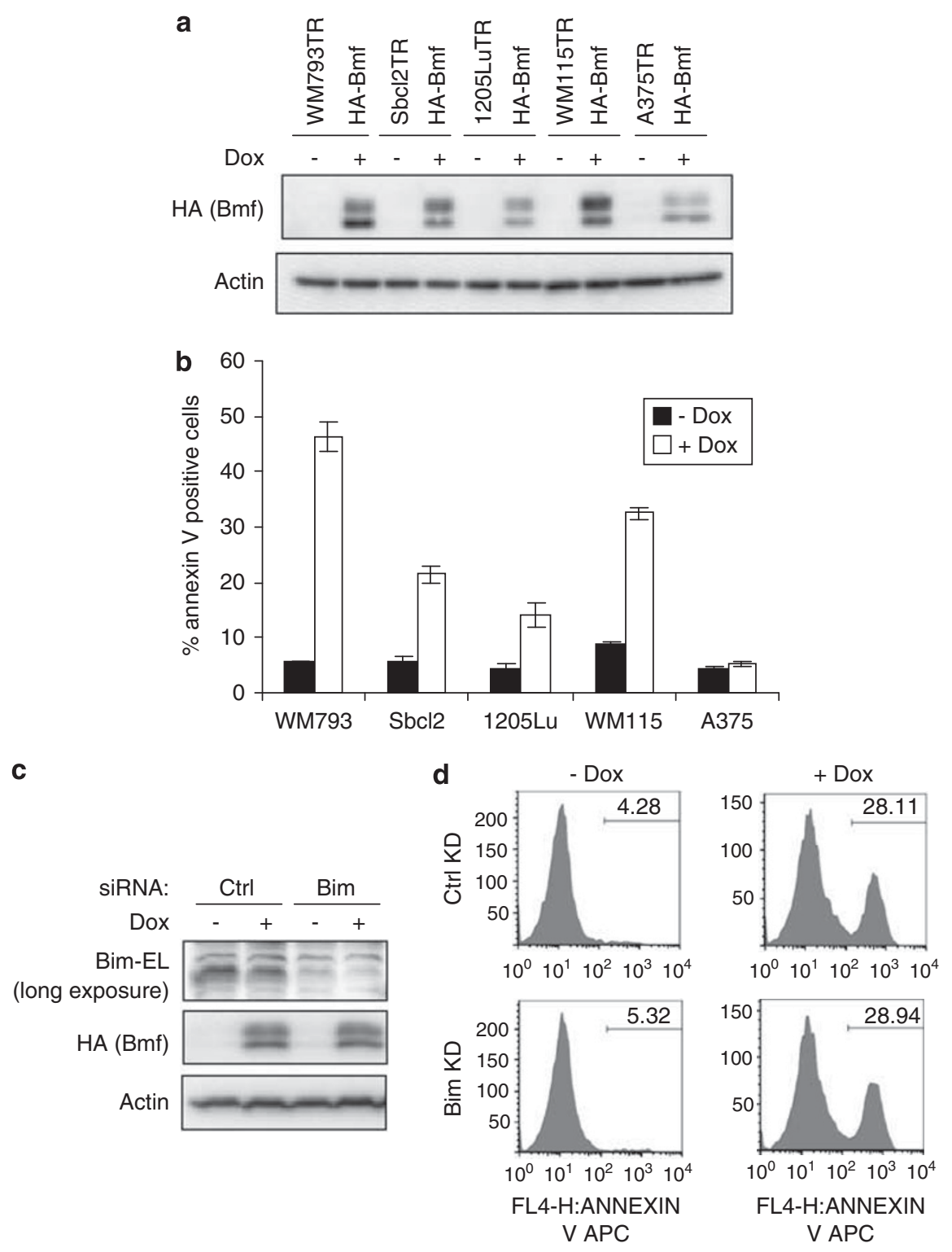

Figure 1 Bmf induces apoptosis in melanoma cells independent of Bim-EL. (a) WM793TR/HA-Bmf, Sbcl2TR/HA-Bmf, 1205LuTR/HA-Bmf, WM115TR/HA-Bmf and A375TR/HA-Bmf cells were treated with or without $100 \mathrm{ng} / \mathrm{ml}$ doxycycline for $7 \mathrm{~h}$. Cell lysates were analyzed by western blotting using HA-tag and actin (loading control) antibodies. (b) As for a, except that cells were induced with or without $100 \mathrm{ng} / \mathrm{ml}$ doxycycline for $48 \mathrm{~h}$. Cells were then stained with annexin-V-APC for flow cytometry analysis. Quantitated are the mean percentages of annexin-V-positive cells from three independent experiments. Error bars: standard deviation. (c) WM793TR/HA-Bmf cells were transfected with control or Bim siRNA for $72 \mathrm{~h}$. Cells were then treated with or without $100 \mathrm{ng} / \mathrm{ml}$ doxycycline for additional $24 \mathrm{~h}$ before lysis for western blot analysis. (d) As in c except that after $24 \mathrm{~h}$ of doxycycline treatment, cells were subject to annexin- $\mathrm{V}$ staining and flow cytometry analysis. Representative flow traces from two independent experiments flow traces are shown. $x$ axis, fluorescence intensity; $y$ axis, cell numbers

sufficient to induce a mobility shift (Figure 3b). Importantly, the effects of alanine replacement of S74 and S77 on the electrophoretic mobility shift of Bmf were recapitulated in other two melanoma cell lines, 1205Lu and A375 (Figure 3c), indicating these post-translational modifications on Bmf are not cell line specific. To further test whether the electrophoretic mobility shift is associated with S74/S77 phosphorylation, we treated Bmf-expressing WM793 cells with the serine/threonine phosphatase inhibitor, okadaic acid. Short-term treatment of this inhibitor increased the level of phospho-MEK and led to accumulation of the slow-migrating population of the WT Bmf (Figure 3d). The okadaic acid induction of slower-migrating forms of Bmf was not observed with the S74A/S77A mutant form, further supporting that Bmf is phosphorylated at S74 and S77 sites.

To test whether ERK2 signaling regulates phosphorylation at these two sites, we knocked down ERK1, ERK2 or p38 $\alpha$ in Dox-treated WM793 HA-Bmf S74A and WM793 HA-Bmf S77A cell lines. Indeed, siRNA-mediated ERK2 depletion efficiently reduced $\mathrm{Bmf}$ mobility shift in both cell lines, whereas ERK1 and $p 38 \alpha$ knockdown showed no effect (Figure 3e), consistent with previous results. Therefore, ERK2 signaling 
a

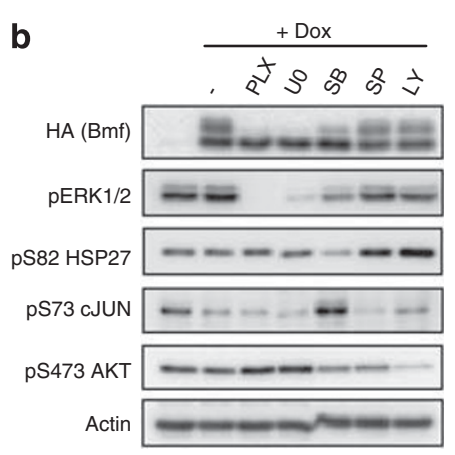

C

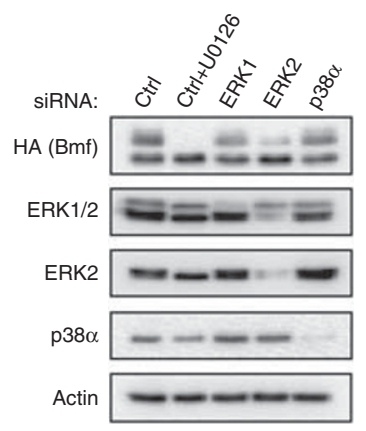

d

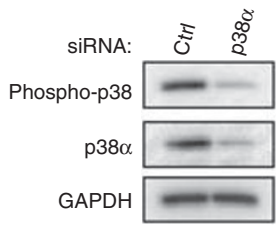

e

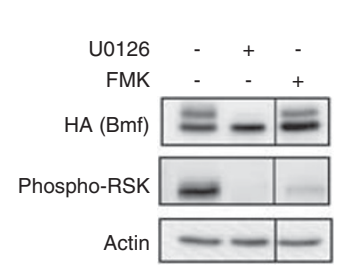

f

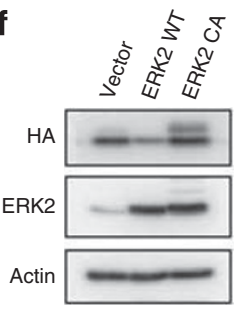

Figure 2 ERK2 signaling regulates Bmf phosphorylation. (a) A375TR/HA-Bmf WT cells were treated with $100 \mathrm{ng} / \mathrm{ml}$ doxycycline overnight and followed by immunoprecipitation using HA-tag antibody. The immunoprecipitates were treated with CIAP or CIAP plus phosphatase inhibitor for $1 \mathrm{~h}$ before western blot analysis. Indicated are the percentages of Bmf that is slow migrating, as determined by quantitation of band intensity. (b) WM793TR/HA-Bmf cells were treated with or without $100 \mathrm{ng} / \mathrm{ml}$ doxycycline and PLX4720, U0126, SB203580, SP600125, LY294002, as indicated for $7 \mathrm{~h}$. Cell lysates were analyzed by western blotting using the antibodies indicated. Actin serves as a loading control. (c) WM793TR/HA-Bmf cells were transfected with control, ERK1, ERK2 and p38 $\alpha$ siRNA. Seventy-two hours post transfection, cells were treated with $100 \mathrm{ng} / \mathrm{ml}$ doxycycline and with or without $10 \mu \mathrm{M}$ U0126 for $7 \mathrm{~h}$ before lysis for western blot analysis. (d) Cells were transfected with control non-targeting or p38 $\alpha$-specific siRNAs for $72 \mathrm{~h}$ before lysis for western blot analysis using phospho-p38, p38 $\alpha$ and GAPDH (loading control) antibodies. (e) WM793TR/HA-Bmf cells were treated with $100 \mathrm{ng} / \mathrm{ml}$ doxycycline plus DMSO, $10 \mu \mathrm{M}$ U0126 or $1 \mu \mathrm{M}$ FMK for $7 \mathrm{~h}$. Lysates were analyzed by western blotting using antibodies against HA-Tag, phospho-RSK and actin (loading control). (f) HA-Bmf was co-expressed with wild-type ERK2 (ERK2 WT) or constitutive active ERK2 (ERK2 CA) or empty vector in 293FT cells. The effect of ERK2 on Bmf mobility shift was examined by western blot analysis

but not ERK1 or $\mathrm{p} 38 \alpha$ signaling regulates Bmf phosphorylation at serine 74 and serine 77 . Next, we performed in vitro protein kinase assays using activated ERK2 and bacterially expressed glutathione $S$ transferase (GST)-Bmf fusion protein variants including GST-Bmf WT, GST-Bmf S74A, GST-Bmf S77A and GST-Bmf AA (Figure 3f). Efficient phosphorylation was detected with WT, S74A GST-Bmf. By contrast, phosphorylation was inefficient in the S77A mutant and not detected in GST-Bmf AA, indicating ERK2 directly phosphorylates both serine 74 and serine 77 in Bmf but that serine 77 is the major site.

Phosphorylation of serine 74 and/or serine 77 does not affect Bmf protein stability. Phosphorylation of BH3-only protein, Bim-EL, by ERK on serine 69 has been shown to promote its targeting by proteasome-dependent degradation. ${ }^{17,25}$ To test whether phosphorylation of serine 74 and/or serine 77 would similarly reduces Bmf protein stability; we treated melanoma cells expressing HA-Bmf with actinomycin $D$ and measured Bmf protein levels at different time points (Figure 4). A time-dependent decrease in the Bmf protein level was observed in all four melanoma cell lines that express WT, S74A, S77A or AA HA-Bmf, respectively. The half-life of Bmf protein is $\sim 4 \mathrm{~h}$ and no apparent difference in the turnover rate was observed among the Bmf variants, suggesting that phosphorylation of serine 74 and/or serine 77 has no influence on Bmf protein stability.

Phosphorylation of Bmf on serine 77 reduces its apoptotic activity. Phosphorylation of $\mathrm{BH} 3-$ only proteins often modulates their pro-apoptotic activities. ${ }^{16,26}$ It has been previously shown that the phosphomimetic S74D mutation of Bmf enhanced its apoptotic activity in vivo. ${ }^{22}$ Therefore, we examined the apoptotic activity of WT Bmf and its alanine or aspartic acid substiution variants in melanoma cells. Expression of WT Bmf in WM793 cells for $16 \mathrm{~h}$ induced apoptosis with $a \sim 25 \%$ of the cells displaying annexin-V positivity (Figure 5a). Replacement of serine 74 with alanine (S74A) only slightly increased Bmf-induced apoptosis, whereas alteration of serine 77 (S77A) alone or together with serine 74 (AA) enhanced apoptosis to a significantly higher level (Figure 5a). These data suggest that inability to phosphorylate $S 77$ augments Bmf's apoptotic activity. Consistent with others' findings, the phosphomimetic S74D Bmf mutant displayed increased apoptosis over WT or S74A Bmf, confirming that phosphorylation of S74 may enhance Bmf apoptotic activity. Conversely, the phosphomimetic S77D mutant restored Bmf-induced cell death to the WT level (Figure 5a). Given the differing effects between S77D and S77A, it is likely that phosphorylation of S77, instead of the $\mathrm{S} 77$ residue itself, reduces Bmf apoptotic activity. Interestingly, the dual aspartic acids replacement mutant (DD) had a higher death rate than the WT or S77D, suggesting that the apoptosis-enhancing effect of S74 phosphorylation counteracts the apoptosis-dampening effect of $S 77$ phosphorylation. Extended Dox treatment (24 and $32 \mathrm{~h}$ ) increased cell death in all Bmf variants but with the same overall pattern, that is, S74A and AA elicited high levels of apoptosis and S77D was equivalent to WT Bmf (Figures 5b and c).

Phosphorylation of Bmf on serine 74 and/or serine 77 does not alter its association with DLC2 or mitochondrial localization. In healthy cells, Bmf is retained in cytoplasm through binding to DLC2. ${ }^{6}$ Proapoptotic signals can cause Bmf to dissociate from DLC2 and translocate to mitochondria. ${ }^{6,14}$ Serine 74 is located within the DLC2-binding domain in Bmf and serine 
a

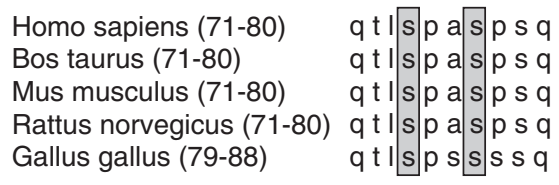

b

WM793TR

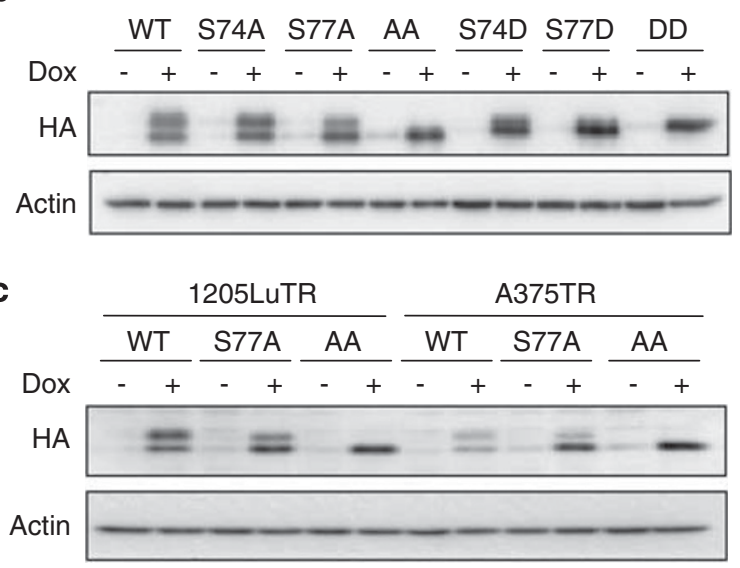

d

\begin{abstract}
HA-Bmf
\end{abstract}
Okadaic Acid

$\mathrm{HA}$ (Bmf)

$\frac{\text { WT }}{-+} \frac{\text { AA }}{-+}$

$\%$ slow migrating:

Phospho-MEK1/2

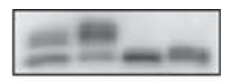

e

$\begin{array}{llll}48 & 75 & 0 & 0\end{array}$

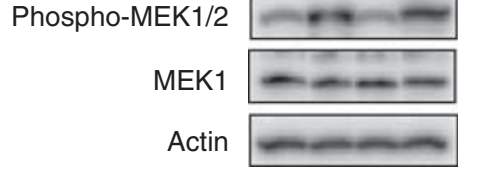

$$
\text { f }
$$
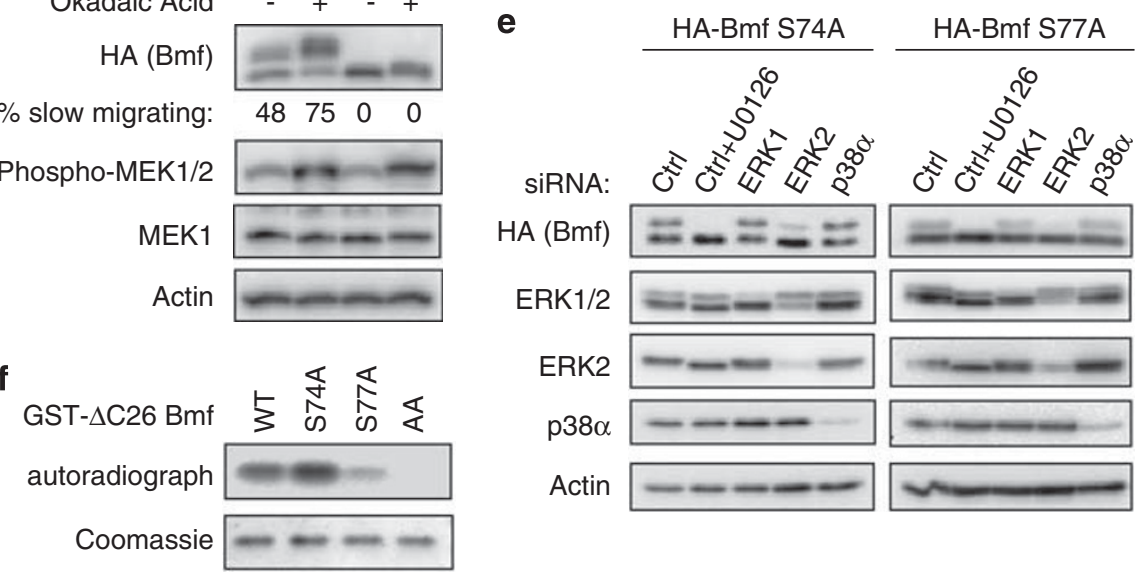

Figure 3 ERK2 directly phosphorylates residue serine 74 and serine 77 in Bmf. (a) Alignment of Bmf protein sequences surrounding serine 74 and serine 77 among different species. Conserved serine 74 and serine 77 residues are shaded. The amino-acid positions relative to the start codon are shown in parentheses. (b) WM793TR cell lines expressing wild type, S74A, S77A, S74A/S77A (AA), S74D, S77D, S74D/S77D (DD) HA-Bmf were induced with with or without $100 \mathrm{ng} / \mathrm{ml}$ doxycycline for 7 h. Cells lysates were analyzed by western blotting using antibodies against the HA-tag and actin. (c) 1205LuTR/HA-Bmf (WT, S77A and AA) cells and A375TR/HA-Bmf (WT, S77A and $\mathrm{AA}$ ) cells were treated with or without $100 \mathrm{ng} / \mathrm{ml}$ doxycycline for $7 \mathrm{~h}$. Cell lysates were analyzed as in $\mathbf{b}$. (d) WM793TR/HA Bmf cells were treated with $100 \mathrm{ng} / \mathrm{ml}$ doxycycline for $1.5 \mathrm{~h}$ and $1 \mu \mathrm{M}$ okadaic acid was added for another $30 \mathrm{~min}$. Cells were then lysed for western blot analysis. Indicated are the percentages of Bmf that is slow migrating, as determined by quantitation of band intensity. (e) WM793TR/HA-Bmf S74A and WM793TR/HA-Bmf S77A cells were transfected with control, ERK1, ERK2 and p38 $\alpha$ siRNA. Seventy-two hours post transfection, cells were treated with $100 \mathrm{ng} / \mathrm{ml}$ doxycycline and with or without $10 \mu \mathrm{M} \cup 0126$ for $7 \mathrm{~h}$ before lysis for western blot analysis on indicated proteins. (f) Activated ERK2 was incubated with bacterially expressed GST-Bmf WT, GST-Bmf S74A, GST-Bmf S77A and GST-Bmf AA in the presence of $\left[\gamma^{32}\right.$ P]ATP. Phosphorylation of GST-Bmf variants was examined by SDS-PAGE followed by autoradiography. Coomassie staining of the same gel is shown below

77 adjacent to it; hence, we hypothesized that phosphorylation of serine 74 and serine 77 may stablize the Bmf association with DLC2 and cause its cytoplasmic retention. To test this notion, we examined the binding of $\mathrm{Bmf}$ mutants to DLC2 in 293FT cells expressing B-RAF ${ }^{\mathrm{V} 600 \mathrm{E}}$ to activate MEK-ERK signaling. Ectopic expression of BRAF $^{\mathrm{V} 600 E}$ led to phosphorylation of Bmf variants (WT, S74A, S77A, AA and DD HA-Bmf) in a manner similar to that in melanoma cells (Figure 6a). However, all Bmf variants associated with DLC2 at a similar level (Figure 6a) indicating that serine 74/77 phosphorylation does not affect Bmf binding to DLC2. We then examined the effect of serine 74/S77 phosphorylation on Bmf subcellular localization. In WM793 cells, Bmf colocalizes with mitochondria as shown by immunofluoresence, and this subcellular localization is not altered by replacement of serine 74 and/or serine 77 with alanine (Figure 6b). Consistently, all Bmf variants (WT, S74A, S77A, AA and DD) were detected in the mitochondriaenriched fraction (Figures $6 \mathrm{c}$ and $\mathrm{d}$ ) and the ratio between the phosphorylated and non-phosphorylated Bmf population 


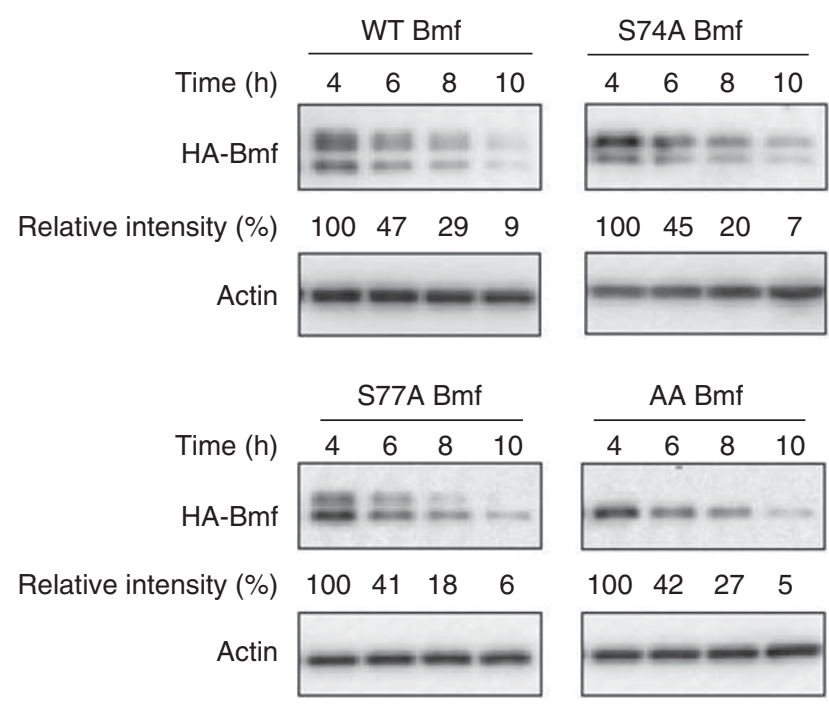

Figure 4 Serine 74 and serine 77 phosphorylation does not affect Bmf protein stability. WM793TR/HA-Bmf cell lines (WT, S74A, S77A and AA) were individually treated with $100 \mathrm{ng} / \mathrm{ml}$ doxycycline. After $1 \mathrm{~h}$, cells were washed in fresh medium and cultured with medium containing $1 \mu \mathrm{g} / \mathrm{ml}$ actinomycin D but without doxycycline for additional $10 \mathrm{~h}$. During this time course, cell samples were removed after 4, 6, 8 and $10 \mathrm{~h}$ for western blot analysis using HA-tag and actin antibodies. The relative intensities of HA-Bmf bands (normalized against actin) were shown below the HA-Bmf blot

was comparable between total lysates and the mitochondria fraction, indicating the status of serine 74 and serine 77 phosphorylation does not affect Bmf translocation to mitochondria.

Phosphorylation of Bmf on serine 74 and/or serine 77 does not affect Bmf association with pro-survival Bcl-2 family proteins. Bmf was known to bind various pro-survival Bcl-2 family proteins including $\mathrm{Mcl}-1, \mathrm{Bcl}-2$ and Bcl-xL. Therefore, we asked whether loss of S74 and/or S77 phosphorylation would affect Bmf interaction with these binding partners. As expected, WT Bmf coimmunoprecipitated with $\mathrm{Bcl}-\mathrm{xL}, \mathrm{Bcl}-2$ and $\mathrm{Mcl}-1$ (Figures $7 \mathrm{a}-\mathrm{C}$ ). However, its binding affinity with these $\mathrm{Bcl}-2$ proteins was not affected by the status of S74 and/or S77 phosphorylation (Figures $7 \mathrm{a}-\mathrm{c}$ ). These data indicate that the apoptotic effects of serine 74 and serine 77 phosphorylation are likely not mediated through altered association with $\mathrm{Bcl}-\mathrm{xL}, \mathrm{Bcl}-2$ and $\mathrm{Mcl}-1$.

\section{Discussion}

BH3-only protein activity is frequently modulated by posttranslational alterations. The effects of phosphorylation on the activity of Bim-EL and Bad and the kinases that are responsible have been studied in depth. ${ }^{13,15-20}$ By contrast, mechanisms that regulate Bmf are poorly understood. Here, we demonstrate that ERK2 phosphorylates serine 74 and serine 77 in Bmf and that serine 77 phosphorylation downregulates Bmf pro-apoptotic function. Serine 74 and serine 77 are highly conserved within Bmf across species (Figure $3 a$ ) but are poorly conserved within other BH3-only proteins. Our findings demonstrate that ERK2 controls Bmf activity via phosphorylation and, thus, regulates Bmf by mechanisms additional to transcript control. ${ }^{11}$

Our study shows a previously unrecognized ability of ERK2 to phosphorylate Bmf. A previous study showed that JNK was able to phosphorylate recombinant Bmf in vitro. ${ }^{13}$ The site of phosphorylation is likely serine 74 based on a similarity to a JNK phosphorylated site, serine 58, in Bim-L. ${ }^{13}$ Our studies were performed in melanoma cells, in which signaling through the ERK1/2 pathway is constitutively elevated due to activating mutations in B-RAF and N-RAS. ${ }^{27,28}$ Although both serine 74 and serine 77 are phosphorylated in Bmf, serine 77 appears to be the major phosphorylation site of the two. Phosphorylation of serine 74 increased Bmf pro-apoptotic activity in melanoma cells, a finding in line with the work of others that was based on decreased numbers of $B$ and $T$ cells in the spleen of Bim-/-, Bmf ${ }^{574 D / S 74 D}$ compared with Bim-/mice. $^{22}$ By contrast, phosphorylation at serine 77 is associated with decreased apoptotic activity in melanoma cells demonstrating functional relevance of this regulation.

Loss of serine 77 phosphorylation in Bmf enhances its proapoptotic function but the mechanism of enhanced activity is less clear. Bmf, similar to Bim-EL, contains a conserved DLCbinding motif. In Bmf, this motif promotes association with DLC2 and the actin cytoskeleton, ${ }^{6}$ whereas the DLC-binding motif in Bim interacts with DLC1 and enhances Bim localization to the microtubule network. JNK phosphorylation at threonine 56 within the DLC motif of Bim-L, dissociated Bim-L from DLC1 and enhanced its apoptotic activity. ${ }^{13} \mathrm{We}$ show that ERK2 phosphorylation of serine 74 (within the DLC motif) and serine 77 (adjacent to the DLC motif) did not alter the association of DLC2 with Bmf. These data complement studies showing that the pro-apoptotic activity of a Bmf mutant that does not bind to DLC2, Bmf A69P, is potentiated by MEK inhibition. ${ }^{14}$ Furthermore, the ability of Bmf to localize to the mitochondria is unaltered by mutation of these phosphorylation sites. In addition, we did not observe that MEK-ERKregulated phosphorylation of Bmf altered its protein turnover. This lack of effect on Bmf turnover is in stark contrast to the action of ERK1/2-mediated phosphorylation of Bim-EL, which promotes ubiquitin-mediated degradation of Bim-EL. The likely underlying reason is that serine 69 in Bim-EL, which is phosphorylated and mediates ERK1/2 effects, is not conserved in Bmf. Finally, no apparent differences were observed in the binding affinities of Bmf with its anti-apoptotic partners, $\mathrm{Bcl}-\mathrm{xL}, \mathrm{Bcl}-2$ or $\mathrm{Mcl}-1$. As all Bmf variants (WT, S74A, S77A and AA) translocate to mitochondria outer membrane shortly after induction (Figures $6 \mathrm{~b}$ and $\mathrm{c}$ ), the effect of $\mathrm{S77}$ phosphorylation is likely mediated through some Bmf-interacting factors within mitochondria.

Our studies relate to cell survival mechanisms in mutant B-RAF melanomas with elevated MEK-ERK1/2 pathway activity. Targeting B-RAF with either the RAF inhibitor PLX4720 or knockdown approaches sensitizes mutant BRAF melanoma cells to apoptosis. ${ }^{11}$ Notably, Bmf is required for this process ${ }^{11}$ although additional input can be provided by Bim-EL, Bim-S and Bad. ${ }^{29-32}$ Here, we show that expression of Bmf is sufficient to induce apoptosis in mutant B-RAF cells and one mutant N-RAS line (Sbcl2). These effects were observed in regular 2D culture conditions and were enhanced in 3D (data not shown). Notably, one line (A375) did not show 
a

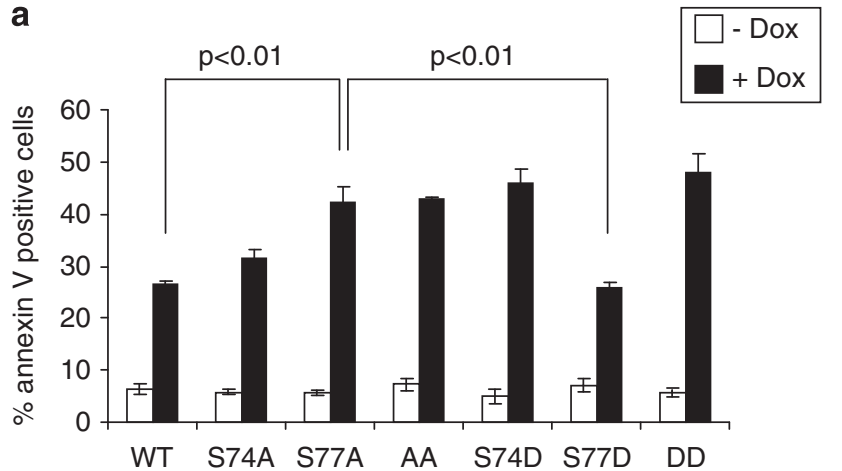

b

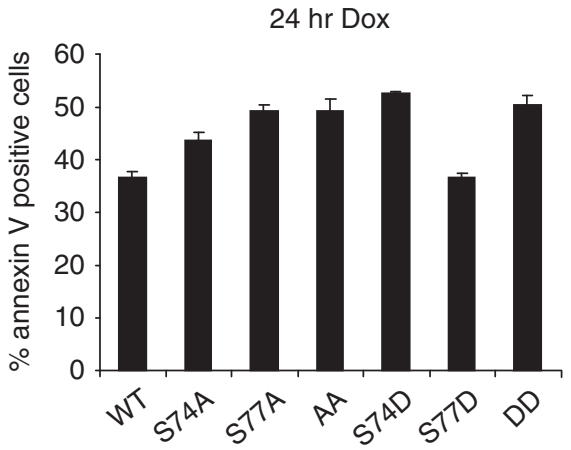

C

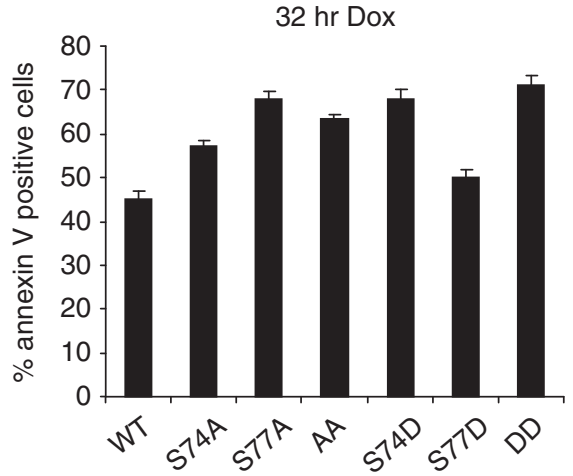

Figure 5 Phosphorylation on serine 77 reduces apoptotic activity of Bmf. (a) WM793TR cell lines engineered to inducibly express HA-Bmf variants (WT, S74A S77A, AA, S74D, S77D and DD) were treated with or without $100 \mathrm{ng} / \mathrm{ml}$ doxycycline for $16 \mathrm{~h}$. Cells were stained with annexin-V-APC and analyzed by flow cytometry. Quantitated are the mean percentages of annexin-V-positive cells from three independent experiments. Error bars: standard deviation. (b) As in a except that cells were treated with $100 \mathrm{ng} / \mathrm{ml}$ doxycycline for $24 \mathrm{~h}$. (c) As in a except that cells were treated with $100 \mathrm{ng} / \mathrm{ml}$ doxycycline for $32 \mathrm{~h}$

enhanced annexin- $V$ staining following Bmf expression. A375 cells display high intrinsic resistance to apoptosis induced by PLX4720, ${ }^{11}$ despite the ability of Bmf to localize to the mitochondria in these cells (data now shown). The mechanisms that act either in a parallel manner or in a downstream of $\mathrm{Bmf}$ mitochondrial localization to promote resistance to $\mathrm{Bmf}$ in A375 cells warrant further investigation, as they may be pertinent to the observed intrinsic resistance to PLX4032 in melanoma patients. ${ }^{33}$ Our data also indicate that 'switching on' Bmf expression alone may not be the most efficient strategy to trigger apoptosis in melanoma cells, as ERK2mediated phosphorylation acts to down-modulate Bmf activity. The development of Bmf-like BH3-mimetics to combine with and enhance the pro-apoptotic activity of PLX4032 should be considered. PLX4032 induces tumor shrinkage in the majority of B-RAF ${ }^{\mathrm{V} 600 \mathrm{E}}$ melanoma patients, indicating that cell death occurs in vivo, but the effects are often short-term (an average of 7-8 months of clinical benefit). ${ }^{33}$ Thus, the need to optimize PLX4032 activity to improve response rates in melanoma is a critical avenue of investigation.

\section{Materials and Methods}

Chemicals and inhibitors. PLX4720 was kindly provided by Dr. Gideon Bollag (Plexxikon Inc., Berkeley, CA, USA). U0126 and LY294002 were purchased from Cell Signaling Technology (Beverly, MA, USA). SB203580, SP600125 and okadaic acid were from Sigma-Aldrich (St. Louis, MO, USA). p90RSK inhibitors, FMK, have been previously described. ${ }^{25}$ Dox, isopropyl $\beta$-D-1thiogalactopyranoside and CIAP were from Fisher Scientific (Pittsburgh, PA, USA).

Cell culture. Melanoma cells were cultured in MCDB 153 containing $20 \%$ Leibovitz L-15 medium, 2\% fetal bovine serum (FBS), $5 \mu \mathrm{g} / \mathrm{ml}$ insulin and penicillin/streptomycin. Human embryonic kidney $293 \mathrm{FT}$ cells were grown in DMEM supplemented with 10\% FBS, 1\% non-essential amino acids and penicillin/ streptomycin

Western blotting. Western blotting was performed as previously described. ${ }^{11}$ Immunoreactivity was detected using peroxidase-conjugated secondary antibodies and chemiluminescence substrate (Pierce, Rockford, IL, USA). Chemiluminescence was visualized using a Versadoc Imaging system (Bio-Rad, Hercules, CA, USA)

Antibodies. The following antibodies were purchased from Cell Signaling Technology: HA-tag (\#2367), p38 $\alpha$ (\#9212), phospho-ERK1/2 (Thr202/Tyr204, \#4377), phospho-p38 (T180/Y182, \#9211), phospho-Akt (Ser473, \#9271), phospho-HSP27 (Ser82, \#2406), phospho-c-JUN (Ser73, \#9164), phospho-RSK (Ser380, \#9341), GAPDH (\#2118). ERK2 (sc-1647) and ERK1/2 (sc-094) antibodies were purchased from Santa Cruz Biotechnology, Inc. (Santa Cruz, CA, USA). Anti-actin (A2066) and anti-FLAG-tag (F3165) antibodies were obtained from Sigma-Aldrich. Anti-Bim (AAP-330) was from Stressgen (San Diego, CA, USA).

siRNA transfection. Cells were transfected with siRNAs at a concentration of $25 \mathrm{nM}$ for $72 \mathrm{~h}$ using Oligofectamine (Invitrogen, Carlsbad, CA, USA), as previously described. ${ }^{34}$ Non-targeting control (5'-UGGUUUACAUGUCGACUAA-3'), ERK1 (5'-GACCGGAUGUUAACCUUUA-3'), ERK2 (5'-CCAAAGCUCUGGACUUAUU-3'), p38 $\alpha\left(5^{\prime}\right.$-GGAAUUCAAUGAUGUGUAU-3') siRNAs were purchased from Dharmacon (Chicago, IL, USA). Bim siRNA (5'-GACCGAGAAGGUAGACAAUUG- $3^{\prime}$ ) was from Cell Signaling Technology.

Annexin-V staining. Staining was performed as described previously ${ }^{11}$ and analyzed by flow cytometry on a FACSCalibur (BD Biosciences, San Jose, CA, USA) in the KCC Flow cytometry shared resource facility. Data were analyzed using Flowjo software (Three Star, Inc., Ashland, OR, USA).

Generation of melanoma cell lines that inducibly express HA-Bmf variants. NH2-terminal HA-tagged WT Bmfs were amplified from human Bmf cDNA plasmid pBabe-HA-human Bmf (Addgene Inc., Cambridge, MA, USA) using the KOD Hot Start DNA polymerase kit (Novagen, Madison, WI, USA) and the primers, forward 5-CACCATGTACCCCTACGACGTGCC-3 and reverse 5-TCACCTAGGGCCTGCCCCGTTCC-3. The HA-Bmf WT PCR fragments were then cloned into $\mathrm{pENTR/D-TOPO}$ vector (Invitrogen) to generate entry plasmid pENTR/HA-Bmf WT. Entry plasmids harboring mutant Bmf alleles, including S74A, S77A, S74A/S77A (AA), S74D and S77D, were constructed using Quickchange site-directed mutagenesis kit (Agilent Techonologies Inc., Santa Clara, CA, USA) and pENTR/HA-Bmf WT as template. Primers used in mutagenesis PCR are available on request. Bmf entry plasmids were individually recombined with a pLenti/ TO/V5-DEST containing the puromycin resistance cassette using the LR Clonase II kit and protocol (Invitrogen) to generate corresponding destination plasmids (pLentipuro/TO/HA-Bmf WT, S74A, S77A, AA, S74D, S77D). Lentiviruses were 

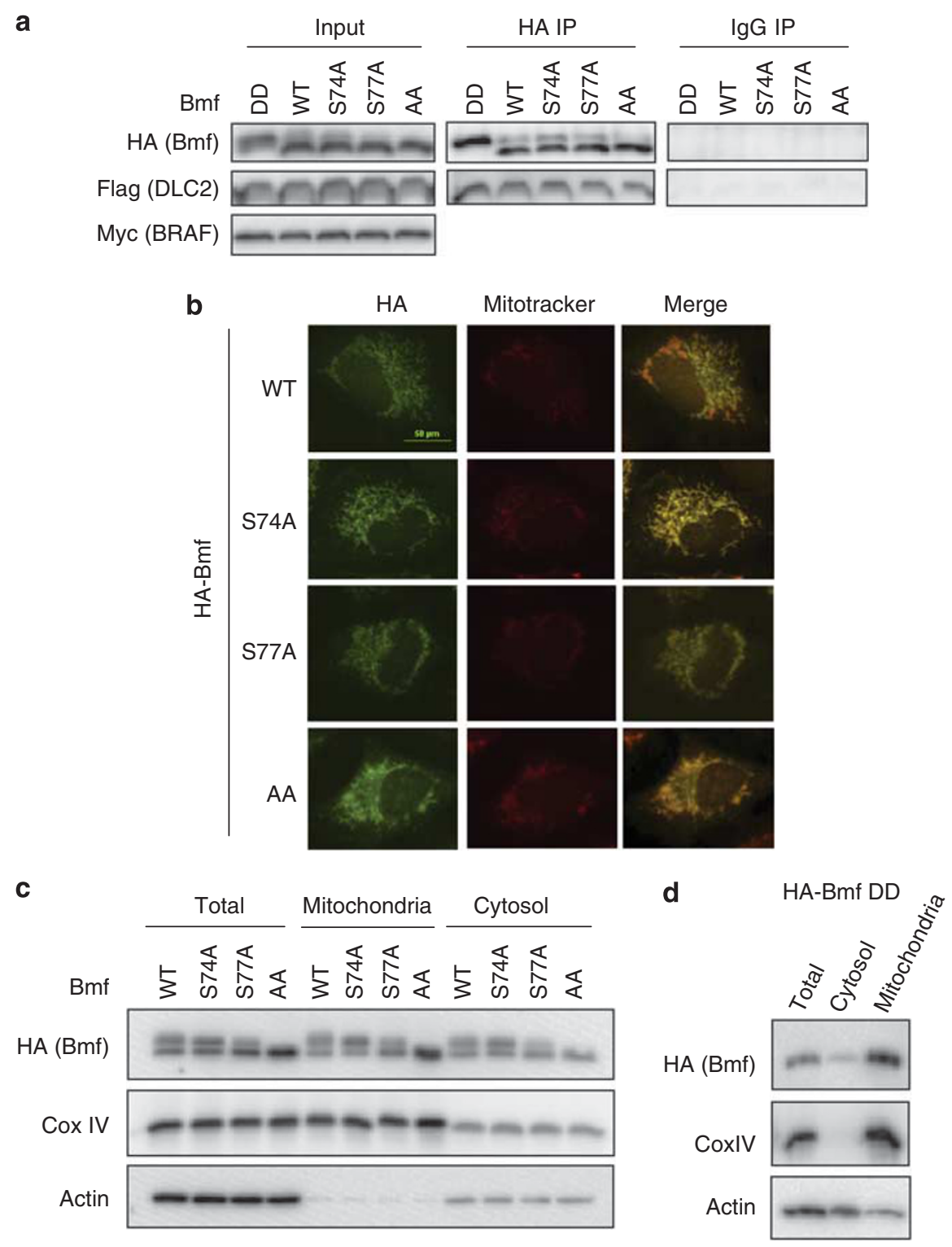

Figure 6 Phosphorylation of serine 74 and serine 77 does not alter Bmf association with DLC2 and localization to the mitochondria. (a) HA-Bmf variants (DD, WT, S74A, S77A and AA) were co-expressed with FLAG-tagged DLC2 and Myc-tagged B-RAF ${ }^{\mathrm{V} 600 \mathrm{E}}$ in $293 \mathrm{FT}$ cells for $24 \mathrm{~h}$. Immunoprecipitation was performed using normal Rabbit IgG or antibody against the HA epitope tag. The presence of HA-Bmf and FLAG-DLC2 in immunoprecipitates and levels of transgene expression in cell lysates (input) was examined by western blot analysis using antibodies against HA and FLAG epitope tags. (b) WM793TR/HA-Bmf WT, S74A, S77A and AA cells were simultaneously treated with $100 \mathrm{ng} / \mathrm{ml}$ doxycycline $(1 \mathrm{~h})$ and $200 \mathrm{nM}$ mitotracker Red CMXRos $(30 \mathrm{~min})$. Cells were then fixed for immunofluoresence analysis. HA-tag antibody was used to detect HA-Bmf (green, left panel). Mitochondria were highlighted by Red CMXRos dye (red, middle panel). The merged images of HA-Bmf and mitochondria staining are shown on the right. (c) Mitochondria fractionation was performed on doxycycline-treated WM793TR/HA-Bmf WT, S74A, S77A and AA cells. Expression of HA-Bmf in total lysates, mitochondria and cytosol fraction was detected by western blot. Actin and Cox IV were used as controls. (d) As in c except WM793TR/HA-Bmf WT DD cells were used

generated using the ViraPower Lentiviral Gateway Expression kit (Invitrogen), as described previously. ${ }^{30}$ WM793, SbCl2, WM115, 1205Lu and A375 cells that stably express tet repressor $\left(\mathrm{TR}^{30}\right)$ were infected with lentivirus for $72 \mathrm{~h}$ before selection with $10 \mu \mathrm{g} / \mathrm{ml}$ puromycin. Expression of the inducible transgene was induced by addition of Dox $(100 \mathrm{ng} / \mathrm{ml})$ to the medium, as previously described. ${ }^{35,36}$

Transient transfection. All transient transfections in 293FT cells were performed using Fugene 6HD (Roche, Indianapolis, IN, USA) according to the manufacturer's guidelines. The plasmids used pCMV5 His-ERK2 WT and pCMV His-ERK L73P/S151D (constitutive active ERK2) were gifts from Dr. Melanie Cobb
(University of Texas Southwestern) and Dr. Natalie Ahn (University of Colorado), respectively. ${ }^{37}$

Construction and purification of GST-HA-Bmf $\Delta$ C26 fusion proteins. HA-Bmf WT and mutant alleles were individually PCR amplified using aforementioned Bmf entry vectors as template and the primers, BamHI Bmf F (5'-CGCGTGGATCCATGTACCCCTACGACGTGC-3') and EcoRI Bmf $\triangle$ C26 R (5'-CGCGTGAATTCTCAACGATTTTGGTCTGCTGGTG-3'). To increase the solubility of GST-HA-Bmf fusion protein, the C-terminal 26 amino acids of Bmf (putative transmembrane domain) was deleted during PCR. ${ }^{38}$ The HA-Bmf $\Delta$ C26 amplicons were digested with $B a m H I$ and $E c o R I$, cloned into pGEX-KG vector 


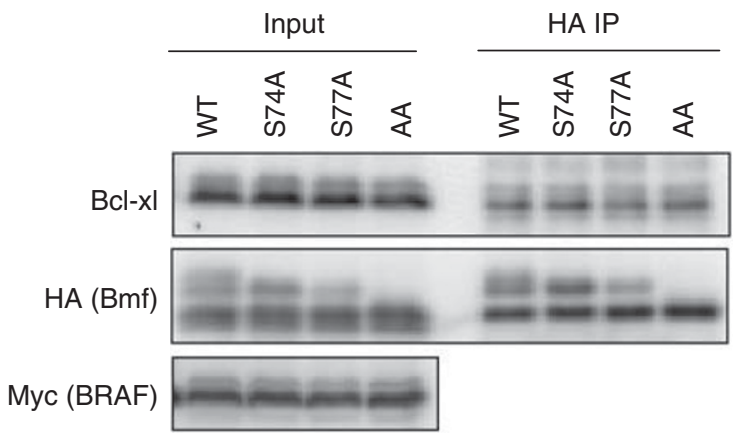

b

Input

HA IP

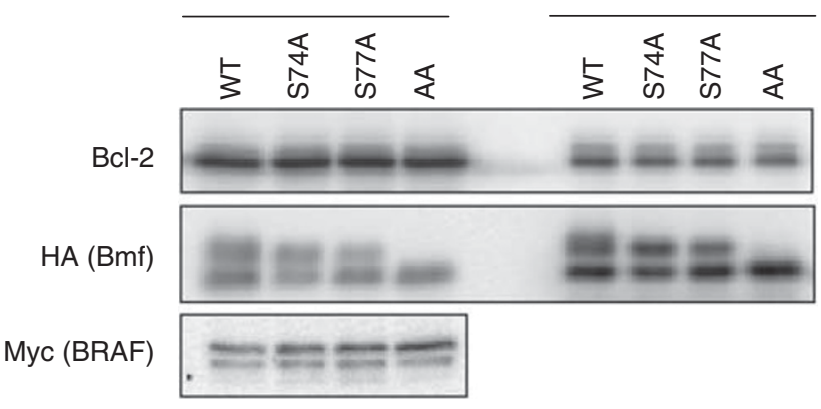

C

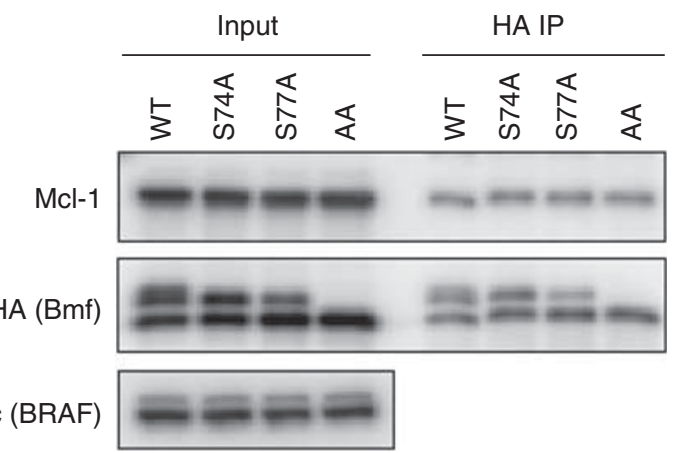

Figure 7 Phosphorylation of serine 74 and serine 77 does not alter Bmf association with pro-survival proteins. HA-Bmf variants (WT, S74A, S77A and AA) were co-expressed in $293 \mathrm{FT}$ cells with Myc-tagged B-RAF ${ }^{\mathrm{V} 600 \mathrm{E}}$ and (a) Bcl-xL, (b) $\mathrm{Bcl}-2$ or (c) Mcl-1 for $24 \mathrm{~h}$. Immunoprecipitation was performed using antibody against the $\mathrm{HA}$ epitope tag. The presence of $\mathrm{Bcl}-\mathrm{xL}, \mathrm{Bcl}-2$ and $\mathrm{Mcl}-1$ in immunoprecipitates (HA IP) and levels of transgene expression in cell lysates (input) was examined by western blot analysis

(Addgene) to form GST-HA-Bmf $\triangle$ C26 fusions and transformed into BL21 (DE3) cells. Bacterial cells harboring GST-HA-Bmf $\Delta \mathrm{C} 26$ fusion constructs were grown to $\mathrm{OD}_{600}=0.5$ and induced with $0.1 \mathrm{M}$ isopropyl $\beta$-D-1-thiogalactopyranoside for $3 \mathrm{~h}$. Cells were pelleted, resuspended in PBS supplemented with protease inhibitors and lysed by addition of $0.5 \%$ triton X-100 followed by sonication. After centrifugation, supernatant was collected and incubated with pre-washed glutathione-agarose beads for $2 \mathrm{~h}$. Next, glutathione S-agarose beads were collected by centrifugation and washed in PBS. GST-HA-Bmf $\Delta$ C26 fusion proteins were eluted from washed beads using elution buffer ( $10 \mathrm{mM}$ reduced glutathione, $50 \mathrm{mM}$ Tris- $\mathrm{HCl}, \mathrm{pH} 8.0,5 \%$ glycerol).

In vitro kinase assay. Purified GST-HA-Bmf $\Delta$ C26 WT or mutants were incubated with $\left[\gamma^{32} \mathrm{P}\right]-A T P$ and activated recombinant ERK2 (New England BioLabs., Ipswich, MA, USA) in NEBuffer (New England BioLabs) at $30^{\circ} \mathrm{C}$ for $30 \mathrm{~min}$. The reaction products were diluted in SDS-PAGE loading buffer, separated on SDS polyacrylamide gel and the gel exposed to film.
Immunoprecipitation. Transfected 293FT cells were lysed in NP40 buffer $(150 \mathrm{mM} \mathrm{NaCl}, 1 \% \mathrm{NP} 40,50 \mathrm{mM}$ Tris- $\mathrm{HCl}, \mathrm{pH} 8.0)$. supplemented with protease inhibitor cocktail (Roche). Cell debris were removed by centrifugation and the supernatant was collected and pre-cleared with protein $A / G$ agarose beads. Pre-cleared lysate was incubated with specific antibodies overnight and subsequently with protein $A / G$ agarose beads for another $2 \mathrm{~h}$. Protein $\mathrm{A} / \mathrm{G}$ beads were pelleted, washed in NP40 buffer and resuspended in Laemmli sample buffer for western blot analysis.

CIAP treatment. A375TR-HA-Bmf WT cells were treated with $100 \mathrm{ng} / \mathrm{ml}$ Dox for $24 \mathrm{~h}$ and lysed in NP40 buffer for immunoprecipitation as described above using HA-tag-specific antibody. The immunoprecipitates were incubated with CIAP only or in combination of phosphatase inhibitor cocktail (Roche) for $1 \mathrm{~h}$ at $30^{\circ} \mathrm{C}$. The samples were then diluted with $4 \times$ SDS sample buffer for western blot analysis.

\section{Conflict of interest}

The authors declare no conflict of interest.

Acknowledgements. This work was supported by grants from the National Institutes of Health (GM067893, CA125103) and American Cancer Society (RSG08-03-01-CSM). This project is also funded, in part, under a Commonwealth University Research Enhancement Program grant with the Pennsylvania Department of Health (AEA). Yongping Shao was supported by a fellowship from Outrun the Sun. The Flow Cytometry Shared Resource facility in the Kimmel Cancer Center is supported by National Cancer Institute Support Grant 1P30CA56036 to the Kimmel Cancer Center.

1. Merino D, Giam M, Hughes PD, Siggs OM, Heger K, O'Reilly LA et al. The role of BH3-only protein Bim extends beyond inhibiting Bcl-2-like prosurvival proteins. J Cell Biol 2009; 186: $355-362$.

2. Certo M, Del Gaizo Moore V, Nishino M, Wei G, Korsmeyer S, Armstrong SA et al. Mitochondria primed by death signals determine cellular addiction to antiapoptotic BCL-2 family members. Cancer Cell 2006; 9: 351-365.

3. Du H, Wolf J, Schafer B, Moldoveanu T, Chipuk JE, Kuwana T. BH3-domains other than Bim and Bid can directly activate BAX/BAK. J Biol Chem 2010; 286: 491-501.

4. Cheng EH, Wei MC, Weiler S, Flavell RA, Mak TW, Lindsten T et al. BCL-2, BCL-X(L) sequester $\mathrm{BH} 3$ domain-only molecules preventing BAX-and BAK-mediated mitochondrial apoptosis. Mol Cell 2001; 8: 705-711.

5. Chipuk JE, Moldoveanu T, Llambi F, Parsons MJ, Green DR. The Bcl-2 family reunion. Mol Cell 2010; 37: 299-310.

6. Puthalakath H, Villunger A, O'Reilly LA, Beaumont JG, Coultas L, Cheney RE et al. Bmf: A proapoptotic BH3-only protein regulated by interaction with the myosin $\mathrm{V}$ actin motor complex, activated by anoikis. Science 2001; 293: 1829-1832.

7. Chen L, Willis SN, Wei A, Smith BJ, Fletcher JI, Hinds MG et al. Differential targeting of prosurvival $\mathrm{BCl}-2$ proteins by their $\mathrm{BH}$-only ligands allows complementary apoptotic function. Mol Cell 2005; 17: 393-403.

8. Kuwana T, Bouchier-Hayes L, Chipuk JE, Bonzon C, Sullivan BA, Green DR et al. $\mathrm{BH} 3$ domains of $\mathrm{BH} 3$-only proteins differentially regulate Bax-mediated mitochondrial membrane permeabilization both directly and indirectly. Mol Cell 2005; 17: 525-535.

9. Labi V, Erlacher M, Kiessling S, ManzI C, Frenzel A, O'Reilly L et al. Loss of the BH3-only protein Bmf impairs B cell homeostasis and accelerates irradiation-induced thymic lymphoma development. J Exp Med 2008; 205: 641-655.

10. Frenzel A, Labi V, Chmelewskij W, Ploner C, Geley S, Fiegl $\mathrm{H}$ et al. Suppression of B-cell lymphomagenesis by the BH3-only proteins Bmf and Bad. Blood 2010; 115: 995-1005.

11. Shao $Y$, Aplin A. Akt3-mediated resistance to apoptosis in B-RAF targeted melanoma cells. Cancer Res 2010; 70: 6670-6681.

12. Schmelzle T, Mailleux AA, Overholtzer M, Carroll JS, Solimini NL, Lightcap ES et al. Functional role and oncogene-regulated expression of the BH3-only factor Bmf in mammary epithelial anoikis and morphogenesis. Proc Natl Acad Sci USA 2007; 104: 3787-3792.

13. Lei K, Davis RJ. JNK phosphorylation of Bim-related members of the Bcl2 family induces Bax-dependent apoptosis. Proc Natl Acad Sci USA 2003; 100: 2432-2437.

14. VanBrocklin MW, Verhaegen M, Soengas MS, Holmen SL. Mitogen-activated protein kinase inhibition induces translocation of Bmf to promote apoptosis in melanoma. Cancer Res 2009; 69: 1985-1994.

15. Marani M, Hancock D, Lopes R, Tenev T, Downward J, Lemoine NR. Role of Bim in the survival pathway induced by Raf in epithelial cells. Oncogene 2004; 23: 2431-2441.

16. Ley R, Ewings KE, Hadfield $\mathrm{K}$, Howes $\mathrm{E}$, Balmanno $\mathrm{K}$, Cook SJ. Extracellular signalregulated kinases $1 / 2$ are serum-stimulated 'Bim(EL) kinases' that bind to the BH3-only 
protein Bim(EL) causing its phosphorylation and turnover. J Biol Chem 2004; 279 : 8837-8847.

17. Luciano F, Jacquel A, Colosetti P, Herrant M, Cagnol S, Pages G et al. Phosphorylation of Bim-EL by Erk1/2 on serine 69 promotes its degradation via the proteasome pathway and regulates its proapoptotic function. Oncogene 2003; 22: 6785-6793.

18. Putcha GV, Le S, Frank S, Besirli CG, Clark K, Chu B et al. JNK-mediated BIM phosphorylation potentiates BAX-dependent apoptosis. Neuron 2003; 38: 899-914.

19. Becker EBE, Howell J, Kodama Y, Barker PA, Bonni A. Characterization of the c-Jun $\mathrm{N}$-terminal kinase-BimEL signaling pathway in neuronal apoptosis. J Neurosci 2004; 24 : 8762-8770.

20. Cai B, Chang SH, Becker EB, Bonni A, Xia Z. p38 MAP kinase mediates apoptosis through phosphorylation of BimEL at Ser-65. J Biol Chem 2006; 281: 25215-25222.

21. Dehan E, Bassermann F, Guardavaccaro D, Vasiliver-Shamis G, Cohen M, Lowes KN et al. betaTrCP- and Rsk1/2-mediated degradation of BimEL inhibits apoptosis. Mol Cell 2009; 33: 109-116.

22. Hubner A, Cavanagh-Kyros J, Rincon M, Flavell RA, Davis RJ. Functional cooperation of the proapoptotic Bcl2 family proteins Bmf and Bim in vivo. Mol Cell Biol 2010; 30: 98-105.

23. Kutuk O, Letai A. Displacement of Bim by Bmf and Puma rather than increase in Bim level mediates paclitaxel-induced apoptosis in breast cancer cells. Cell Death Differ 2010; 10: 1624-1635.

24. Cohen MS, Zhang C, Shokat KM, Taunton J. Structural bioinformatics-based design of selective, irreversible kinase inhibitors. Science 2005; 308: 1318-1321.

25. Ley R, Balmanno K, Hadfield K, Weston C, Cook SJ. Activation of the ERK1/2 signaling pathway promotes phosphorylation and proteasome-dependent degradation of the BH3-only protein, Bim. J Biol Chem 2003; 278: 18811-18816.

26. Bonni A, Brunet A, West AE, Datta SR, Takasu MA, Greenberg ME. Cell survival promoted by the Ras-MAPK signaling pathway by transcription-dependent and -independent mechanisms. Science 1999; 286: 1358-1362.

27. Satyamoorthy K, Li G, Gerrero MR, Brose MS, Volpe P, Weber BL et al. Constitutive mitogen-activated protein kinase activation in melanoma is mediated by both BRAF mutations and autocrine growth factor stimulation. Cancer Res 2003; 63: 756-759.

28. Conner SR, Scott G, Aplin AE. Adhesion-dependent activation of the ERK1/2 cascade is by-passed in melanoma cells. J Biol Chem 2003; 278: 34548-34554.
29. Sheridan C, Brumatti G, Martin SJ. Oncogenic B-RafV600E inhibits apoptosis and promotes ERK-dependent inactivation of Bad and Bim. J Biol Chem 2008; 283 22128-22135.

30. Boisvert-Adamo K, Aplin AE. Mutant B-RAF mediates resistance to anoikis via Bad and Bim. Oncogene 2008; 27: 3301-3312.

31. Cartlidge RA, Thomas GR, Cagnol S, Jong KA, Molton SA, Finch AJ et al. Oncogenic BRAF(V600E) inhibits BIM expression to promote melanoma cell survival. Pigment Cell Melanoma Res 2008; 21: 534-544.

32. Jiang CC, Lai F, Tay KH, Croft A, Rizos H, Becker TM et al. Apoptosis of human melanoma cells induced by inhibition of B-RAFV600E involves preferential splicing of bimS. Cell Death Dis 2010; 1: e69.

33. Flaherty KT, Puzanov I, Kim KB, Ribas A, McArthur GA, Sosman JA et al. Inhibition of mutated, activated BRAF in metastatic melanoma. New Eng J Med 2010; 363: 809-819.

34. Boisvert-Adamo K, Aplin AE. B-RAF and PI-3 kinase signaling protect melanoma cells from anoikis. Oncogene 2006; 25: 4848-4856.

35. Boisvert-Adamo K, Longmate W, Abel EV, Aplin AE. Mcl-1 is required for melanoma resistance to anoikis. Mol Cancer Res 2009; 7: 549-556.

36. Hu R, Aplin AE. alphaB-crystallin is mutant B-RAF regulated and contributes to cyclin D1 turnover in melanocytic cells. Pigment Cell Melanoma Res 2010; 23: 201-209.

37. Emrick MA, Hoofnagle AN, Miller AS, Ten Eyck LF, Ahn NG. Constitutive activation of extracellular signal-regulated kinase 2 by synergistic point mutations. J Biol Chem 2001 276: 46469-46479.

38. Hinds MG, Smits C, Fredericks-Short R, Risk JM, Bailey M, Huang DC et al. Bim, Bad and Bmf: intrinsically unstructured BH3-only proteins that undergo a localized conformationa change upon binding to prosurvival Bcl-2 targets. Cell Death Differ 2007; 14: 128-136.

Cell Death and Disease is an open-access journal published by Nature Publishing Group. This work is licensed under the Creative Commons Attribution-Noncommercial-No Derivative Works 3.0 Unported License. To view a copy of this license, visit http://creativecommons.org/licenses/by-nc-nd/3.0/ 